\title{
Redes sociais na produção científica: uma análise da Revista ADMpg Gestão Estratégica
}

Flávio José de Melo ${ }^{1}$

Marcielle Anzilago ${ }^{2}$

Resumo: A utilização de redes sociais vem crescendo ao longo do tempo nas instituições e entre pesquisadores de diversas áreas do conhecimento. O impacto sobre a vida social e a troca de conhecimento na produção cientifica levam à integração desses atores, estreitando laços e estimulando pesquisas. O objetivo deste estudo foi verificar a formação de redes sociais na produção científica da Revista ADMpg Gestão Estratégica. A população da pesquisa compreendeu os artigos publicados no período entre 2010 e 2014. Os testes para verificar as ligações de redes sociais adotados neste estudo foram realizados por meio dos softwares Sociais Network Analysis UCINET 6.531, Network Visualization Program - Netdraw 2.153, Wordlenet e Microsoft Office Excel 2010. Os resultados demonstram que as instituições com maior grau de centralidade foram: a Universidade Tecnológica Federal do Paraná (UTFPR), a Universidade Federal do Paraná (UFPR), seguida pela Universidade Estadual do Oeste do Paraná (UNIOESTE). Essas universidades ocupam papel de destaque na inter-relação e na intermediação dos autores, criando laços com outras instituições para cooperação e difusão do conhecimento. O período com maior interação dos coautores foi o ano de 2012. Entretanto, tais implicações levam a concluir que a cooperação entre os atores dessa rede expõe fracas ligações e conexões esparsas, decorrentes do restrito número de ligações e de grupos isolados de agentes.

Palavras-chave: Produção científica. Redes de coautoria. Redes sociais.

Abstract: The use of social networks has grown over time between researchers from various fields of knowledge and institutions. The impact on social life and the exchange of knowledge in scientific production leads to integration of these actors, strengthening ties and stimulating further research. The aim of this study was to verify the formation of social networks in the scientific production of the Revista ADMpg - Gestão Estratégica. The population of the research included articles published in the Journal ADMpg in the period between 2010 to 2014. The tests to verify the social network connections adopted in this study were carried out through the social software Network Analysis - UCINET 6.531, Network Visualization Program - Netdraw 2153, Wordlenet and Microsoft Office Excel 2010. the results showed that the institutions with the highest degree of centrality were the Universidade Tecnológica Federal do Paraná - UTFPR, Universidade Federal do Paraná - UFPR, followed by the State Universidade Estadual do Oeste do Paraná - UNIOESTE. These institutions have a prominent role in the interrelationship and intermediary between authors, creating links with other institutions for cooperation and dissemination of knowledge. The period with greater interaction between the co-authors took place in 2012. However, these implications lead to the conclusion that cooperation among actors in this network exposes weak connections and sparse connections, due to the restricted number of connections and isolated groups among agents.

Keywords: Scientific production. Networks co-authorship. Social networks.

\footnotetext{
${ }^{1}$ Mestre em contabilidade pela Universidade Federal do Paraná (UFPR). Professor da Faculdade de Ilhéus (CESUPI). Email: f.j.melo@uol.com.br.

${ }^{2}$ Doutoranda em contabilidade pela Universidade Federal de Santa Catarina (UFSC). E-mail: marcianzilago@gmail.com.
}

Página 155 Caderno de Ciências Sociais Aplicadas, Vitória da Conquista/BA, vol. 15, n 25, ano 15, p. 155-177, jan/jun 2018. 


\section{Introdução}

As relações nas redes sociais vêm sendo, cada vez mais, objeto de estudos em reuniões e publicações científicas. A ligação dos atores tem sido objeto de pesquisa de diversas áreas do conhecimento. A forma como esses pesquisadores se vinculam desperta interesse, não apenas da comunidade acadêmica, mas do público em geral. As análises de redes centram-se nas relações entre os atores de determinado grupo. As estruturas que compõem as ligações entre atores de uma rede são definidas por nós e laços, e tal estrutura de conexão, na qual o ator se incorpora, é descrita por essas relações e não apenas por seus atributos (HANNEMAN e RIDDLE, 2005).

Segundo Marteleto (2001), o conceito de rede Network deriva, entre outros significados, de alguns preceitos e relações: sistema de nodos e elos, apoio das conexões entre pessoas ou instituições e outros sistemas. Essas relações podem ocorrer nos mais diferentes níveis de estrutura e organização, representadas por um conjunto de participantes autônomos, unidos por ideias, valores e interesses compartilhados.

Tomaél, Alcará e Di Chiara (2005) chamam atenção para o compartilhamento da informação e do conhecimento, estratégias utilizadas pelas conexões em redes sociais para fortalecer os interesses dos integrantes. Os atores delineiam e ampliam sua rede, conforme sua trajetória e inserção na realidade social. O agrupamento com seus semelhantes estabelece relações de trabalho, amizade e interesses em comum.

Wasserman e Faust (1994) destacam pontos relevantes que devem ser considerados na análise de redes sociais. Entre esses pontos estão as relações entre os atores, canais de transferência de conhecimento ou fluxo de recursos, materiais ou imateriais, cujas ações são consideradas interdependentes em vez de independentes ou unidades autônomas.

Conforme o princípio básico da análise de redes sociais, a estrutura das relações determina o conteúdo das conexões. Os pressupostos teóricos das relações entre redes rejeitam a noção de que as pessoas são combinações de atributos ou de que as instituições são entidades estáticas, com limites claramente definidos. Nessa conjectura a análise é, em tese, aplicável virtualmente a qualquer assunto empírico. Três áreas têm merecido atenção especial dos analistas de redes: os efeitos da centralidade dos agentes sobre o comportamento; a identificação de subgrupos da rede; e a natureza das relações entre as organizações (MIZRUCHI, 2006).

Página 156 Caderno de Ciências Sociais Aplicadas, Vitória da Conquista/BA, vol. 15, n² 25, ano 15, p. 155-177, jan/jun 2018. 
Os conceitos inerentes à concepção das ligações sociais presentes nas redes de coautorias também podem contribuir para uma melhor compreensão de como as Instituições de Ensino Superior (IES) interagem na produção acadêmica e na formação de grupos de estudo. A Revista ADMpg Gestão e Estratégia é um periódico de caráter acadêmico cujo propósito é reunir e promover a interação de diversos agentes nas áreas de administração e correlatas, incentivando a divulgação do conhecimento entre os pesquisadores. Nesse contexto, surge a questão de pesquisa deste estudo, que busca saber: quais são as redes sociais institucionais formadas na revista ADMpg Gestão Estratégica? O objetivo do estudo foi verificar a formação de redes sociais na produção científica da revista ADMpg Gestão Estratégica no período entre 2010 e 2014, bem como a formação de laços de coautorias na estrutura dessa rede.

Esse tipo de estudo se justifica tendo em vista a existência de lacunas estruturais observadas em outras análises que abordam a temática sobre redes sociais. Ademais, a pesquisa pode servir de subsídio para incentivar pesquisadores de instituições distintas na troca e disseminação do conhecimento científico.

O artigo divide-se em seis seções: primeiramente, uma breve introdução, seguida do referencial teórico, que aborda aspectos referentes às relações nas redes sociais, procedimentos metodológicos para as análises empíricas, resultados e discussão, considerações finais e referências.

\section{Referencial teórico}

A plataforma teórica deste estudo ampara-se nos pressupostos da Análise de Redes Sociais, vinculados aos seguintes temas: Reflexões teóricas à luz da Social networks e coautorias em Ciências Sociais.

\subsection{Social networks e coautorias em Ciências Sociais}

O interesse de pesquisadores pela análise de redes sociais tem crescido nos últimos anos. Esse tipo de estudo vem acompanhado de ferramentas e técnicas de análises sofisticadas. O universo das pesquisas em rede proporciona um maior entendimento dos atores de cada grupo, facilita as relações, suas propriedades e outros aspectos fundamentais, fator que contribui para enriquecer as pesquisas

Página 157 Caderno de Ciências Sociais Aplicadas, Vitória da Conquista/BA, vol. 15, n 25, ano 15, p. 155-177, jan/jun 2018. 
nessa área do conhecimento (CARRINGTON, SCOTT e WASSERMAN, 2005; MACHADO, IPIRANGA e MATOS, 2013).

Em 1920, os primeiros estudos de rede voltados para as Ciências Sociais são remetidos à área da Psicologia Social (FREEMAN, 1996). Segundo Martes et al. (2006), especificamente no âmbito dos estudos organizacionais, o estudo sobre Redes Sociais emergiu no final dos anos 1970, quando Williamson (1975) e Aldrich (1979) passaram a observar a forma de relacionamento interorganizacional. No Brasil, as primeiras evidências de utilização de técnicas das redes sociais, na ciência administrativa, estão no artigo de Reed Nelson, publicado na Revista de Administração de Empresas (RAE), em 1984.

No que tange aos estudos dos papéis e posições dos atores nas redes sociais, ganham destaque na literatura autores como Wasserman e Faust (1994), Freeman (1996), Scott (2000) e Everett e Borgatti (2005). Na opinião de Wasserman e Faust (1994), o conceito de posição refere-se a um grupo de atores que estão imersos em uma rede de relações de maneira similar. O de papel diz respeito às características das relações entre os atores e suas posições.

Ao longo dos anos, a análise das redes sociais tornou-se um instrumento influente para estudar os padrões de relacionamentos. A base da aplicação do conceito são as categorias: a) medidas estruturais, b) papéis e posições, e c) análise estatística dos relacionamentos (WASSERMANN e FAUST, 1994; FREEMAN, 1996).

Conforme Viana e Baldi (2008), os nós de uma rede podem ser representados por indivíduos ou por grupos de indivíduos e o conceito, a ele aplicado, flexível, dado que a análise de redes sociais pode ser usada tanto para estudar fenômenos organizacionais como interorganizacionais. Em nível organizacional, a análise de rede descreve os relacionamentos entre os atores ou grupos de indivíduos; em nível interorganizacional, o objetivo é investigar a dinâmica nas relações das organizações em redes horizontais e verticais.

Brito (2002) destaca que, nas análises de redes sociais, quatro elementos morfológicos devem ser considerados na estrutura das redes: os fluxos, os nós, as posições e as ligações.

As características dos elementos morfológicos apresentam-se da seguinte forma: os nós podem ser descritos como um conjunto de agentes, objetos ou eventos presentes na rede, associado ao conceito de pontos focais ou nós que compõem a estrutura. A posição de um ator na rede é definida de acordo com o conjunto das relações estabelecidas com os demais atores do grupo. As ligações ou conexões determinam o grau de difusão ou a densidade dos membros da rede, e os fluxos estabelecem a comunicação, por onde fluem recursos e informações (MALAGOLLI e PAULILLO, 2013).

Página 158 Caderno de Ciências Sociais Aplicadas, Vitória da Conquista/BA, vol. 15, n 25, ano 15, p. 155-177, jan/jun 2018. 
As redes sociais são uma estrutura social composta por um grupo de colaboração e pode ser representada por um gráfico. Com esse arcabouço, cada participante na condição de colaborador ou de representante do grupo deve ser chamado de ator e considerado um nó na teoria dos grafos. As relações entre os atores de uma rede de cooperação configuram links ou laços que permitem visualizar sua direção correspondente aos nós observados de um grupo (ZHANG, 2010).

Pryke (2012) destaca que o principal interesse na análise de redes sociais está no papel ou função do ator em determinada conjuntura. Essa configuração de rede envolve as relações entre dois atores, três ou mais indivíduos presentes no grupo. Na estrutura, observa-se a formação de díades "relação que se estabelece entre dois atores", de tríades "ligação observadas que corresponde a ligação entre três atores" ou grupos com mais de três atores.

As redes sociais podem ser desenvolvidas em qualquer ambiente. No campo acadêmico, elas ocorrem por meio da cooperação entre estudantes, com o intuito de disseminar o conhecimento científico. Nesse cenário, o estabelecimento de rede entre programas de pós-graduação pode gerar uma condição favorável para o aumento do número e melhoria da qualidade da produção científica no país (GUIMARÃES, et al., 2009; NASCIMENTO e BEUREN, 2011).

A proposta é que sejam realizadas abordagens inovadoras e feitos contrapontos com o que diz a literatura sobre as analises de redes sociais. O ideal é que os estudos não se limitem a destacar os atores centrais, mas, sobretudo, as metodologias dominantes e formas alternativas de unificar novos grupos de pesquisas para a produção científica em administração. Essas abordagens ajudam a evidenciar gaps para a formulação de políticas de ações que instiguem a produção de conhecimentos (RIBEIRO, 2015).

\section{Procedimentos metodológicos}

Quanto à abordagem metodológica, esta pesquisa é documental e quantitativa. A análise documental é o processo de levantamento, verificação e interpretação de documentos, com o objetivo de extrair informações para a compreensão de determinado fenômeno. Tal metodologia utiliza técnicas de apreensão, compreensão e análise de documentos. No que se refere ao tratamento e desenvolvimento das informações, a pesquisa documental aproxima-se da pesquisa bibliográfica. Entretanto, as diferenças entre essas duas técnicas referem-se à natureza de determinadas fontes de dados, tais como: diários, biografias, reportagens etc. (MOREIRA, 2005; GIL, 2008; FLICK, 2004). Segundo Cooper e Schindler (2011), a pesquisa quantitativa tenta fazer uma mensuração precisa de

Página 159 Caderno de Ciências Sociais Aplicadas, Vitória da Conquista/BA, vol. 15, n 25, ano 15, p. 155-177, jan/jun 2018. 
algo. Nas pesquisas em administração, o alvo normalmente são as métricas para evidenciar comportamento, conhecimento, opiniões ou atitudes.

\subsection{População e amostra}

A população desta pesquisa compreendeu inicialmente 144 artigos científicos disponíveis na Revista ADMpg Gestão Estratégica, no período entre 2010 e 2014. Segundo Bastos, Zago e Recuero (2016), a vantagem de utilizar banco de dados digitais para a análise de redes sociais está na colaboração entre pesquisas acadêmicas e na identificação de coautorias de maneira relativamente fácil e com indícios sólidos. Outro benefício é o fato de as bases de dados poderem ser convertidas em matrizes numéricas que subsidiam a elaboração dos grafos. O critério para a seleção da amostra foi a interação dos autores (díades, tríades ou mais de três autores). Desses 144 artigos, 91 foram publicados com um único autor, ou "nós soltos", o que resultou em uma amostra final com 53 artigos.

\subsection{Análises de dados}

A análise dos dados foi realizada sob duas perspectivas: primeiro, verificar quais as temáticas de estudo escolhidas pelos autores, bem como a análise descritiva dos dados. Segundo, apresentar as principais medidas de centralidade, o grau de intermediação e sociograma, com o auxílio dos softwares: Sociais Network Analysis - Ucinet 6.531, Netdraw- Network Visualization Program 2.153 e o Wordlenet "utilizado para verificar a frequência de ocorrência de palavras em nuvem”. O critério para essa análise foi a maior frequência de palavras no título, no resumo e nas palavras-chave. Além disso, o Microsoft Office Excel 2010 foi usado para exames complementares.

O Ucinet é um software que inclui vários instrumentos analíticos, com um desempenho altamente eficiente, visto que explora e examina dados para estruturas de análises de redes sociais. Esse pacote fornece ferramentas necessárias para avaliar dados analíticos de medida de centralidade, densidade, entre outros. Além disso, o software traz rotinas para a apreciação de matriz, tais como: álgebra matricial e estatística multivariada. O Netdraw permite a visualização de dados por meio de sociograma, para análise de redes sociais. Tal programa lê arquivos nos formatos Ucinet, DL, Pajek e VNA. Salvar rede e dados de atributos em conjunto, com informações de layout como cores ou coordenadas espaciais constitui outra característica desse software (APOSTOLATO, 2013).

Página 160 Caderno de Ciências Sociais Aplicadas, Vitória da Conquista/BA, vol. 15, n 25, ano 15, p. 155-177, jan/jun 2018. 
Correll, Alexander e Gleicher (2013) destacam que algumas ferramentas de visualização textuais tentam transmitir padrões e tendências. Entretanto, uma das formas mais avançadas de expor nuvens de palavras está no Wordle, tendo em vista uma melhor legibilidade por meio de uma cuidadosa tipografia, em detrimento de uma disposição espacial, apresentada em contrastes e com etiquetas de texto que podem aparecer com cor, grifo ou outro sinal. A aplicação Wordle facilita a análise, pois a visualização lista as palavras dimensionadas pela sua ocorrência relativa, ou seja, quanto mais frequentes em um texto, maior a representação das palavras.

Segundo Mcnaugh e Lam (2010) o Wordle demonstra ser particularmente útil para estudos que abrangem análises quantitativas, ligados às temáticas que envolvem texto falado ou escrito e que podem ser utilizados para as análises preliminares. Nesse aspecto, os principais pontos de interesse nas análises preliminares tendem a confirmar resultados e interpretações subjacentes às análises ou proporcionar um suporte adicional a outras ferramentas analíticas.

\section{Resultados}

\subsection{Análise descritiva}

A análise deste estudo utilizou a técnica bibliométrica. A operacionalização dos dados foi realizada em duas etapas: a análise descritiva, com a técnica da nuvem de palavras e medidas de dispersão, seguida da análise do grau de centralidade de Freeman, grau de intermediação e elaboração do Sociograma. A imagem obtida com a técnica da nuvem de palavras e o auxílio do software Wordlenet aponta, conforme os dados quantitativos, os principais termos utilizados pelos autores nos artigos publicados na Revista ADMpg Gestão e Estratégia. Após essa análise, foram verificados os aspectos ligados à formação de rede de cooperação entre coautorias e instituições.

Weiwei Cui et al. (2010) destacam que, nos últimos anos, com a evolução tecnológica em ascendência, houve uma variação na forma de mostrar resultados de pesquisas acadêmicas. A apresentação de conteúdos por meio da técnica da nuvem tem sido amplamente utilizada para fornecer uma visão geral do conteúdo, e de forma compacta, de um conjunto de documentos em análises textuais. Trata-se de um arranjo retangular em ordem alfabética, classificada pela relevância em um layout sequencial de linha a linha.

Página 161 Caderno de Ciências Sociais Aplicadas, Vitória da Conquista/BA, vol. 15, n 25, ano 15, p. 155-177, jan/jun 2018. 
A exposição de dados em forma de nuvem é utilizada para categorizar e exibir imagens textuais de títulos e outras etiquetas ou rótulos que possibilitem uma classificação hierárquica e quantitativa. A exibição do conteúdo em nuvem considera que a representação das palavras (visual maior ou menor) dependerá da frequência de determinado termo. O tamanho da fonte 'será proporcional ao número de ocorrências observado na análise textual (LEMOS, 2016).

Nas análises textuais, as nuvens são representações visuais de um conjunto de palavras em que atributos de um texto, como as variáveis, tamanho, peso ou cores, por exemplo, são usadas para representar as características de determinado grupo de termos associados. Nesse método de análise, usam-se dois tipos de recursos para criar uma nuvem: as características textuais e o posicionamento das palavras. Nas características textuais, são considerados o peso das cores e o tamanho da fonte, para representar a quantidade de frequência de um item no texto. A disposição das palavras pode ser: por ordem alfabética (Clustering), classificação semântica, especificação de preferências de agrupamentos ou disposição espacial (RIVADENEIRA, et al., 2007).

Neste estudo, foi considerada a frequência de palavras que aparecem nos títulos dos artigos, nos resumos e nas palavras-chaves. A Figura 1 traz os principais temas abordados e publicados na Revista ADMpg Gestão e Estratégia. Nesta análise, os resultados foram interpretados com base no contexto adjacente aos estudos de análise textuais, ou seja, de acordo com a seguinte determinação: quanto maior a dimensão das palavras, maior a quantidade de termos no texto. Quanto ao número de ocorrências, as palavras mais citadas nos textos referentes à rede de cooperação foram: pesquisa, empresas, estudo, objetivo e sustentabilidade. A visualização dessa nuvem forneceu indícios sobre as principais temáticas e tendências que norteiam as pesquisas no âmbito das Ciências Sociais Aplicadas entre 2010 e 2104. A frequente ocorrência do tema sustentabilidade chama atenção, tendo em vista que o periódico constitui um espaço de divulgação para diversas áreas, como logística, marketing, recursos humanos, finanças e correlatas.

Página 162 Caderno de Ciências Sociais Aplicadas, Vitória da Conquista/BA, vol. 15, n 25, ano 15, p. 155-177, jan/jun 2018. 


\section{- Cadernos de CIIENCIAS SOCIAIS APLICADAS}

Figura 1: Nuvem de palavras com base nos títulos dos artigos considerados nesta pesquisa.

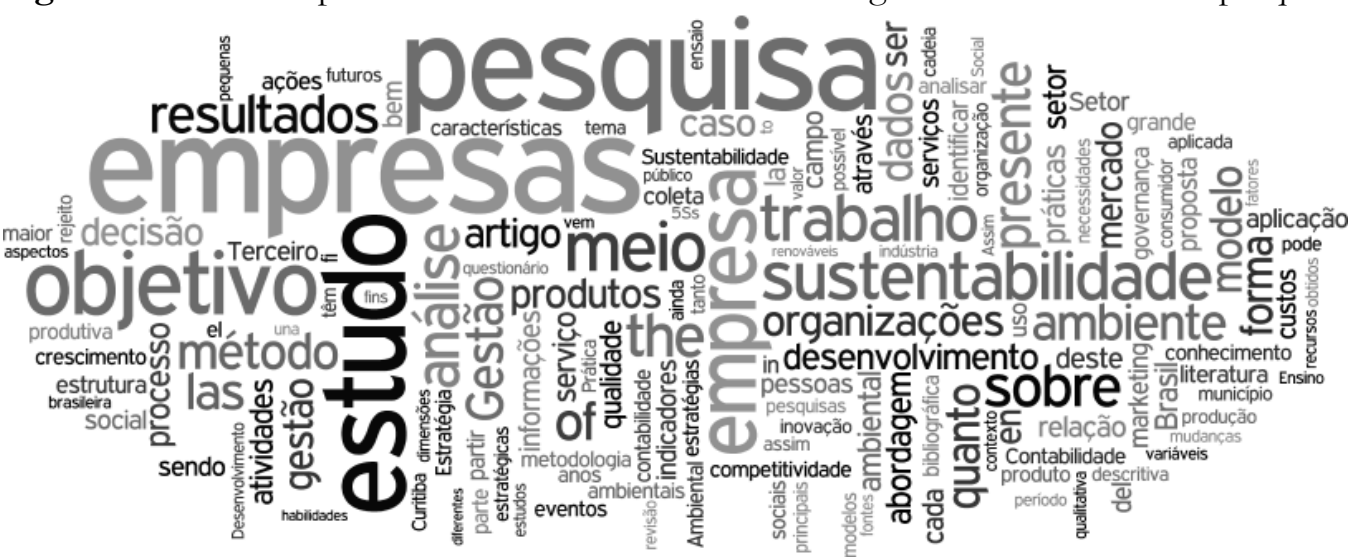

Fonte: Dados da pesquisa (2015).

Os artigos analisados evidenciam a formação de pequenas redes de cooperação entre os autores. A análise estatística descritiva para verificação da frequência de ligações entre os autores e suas respectivas instituições de origem revelou que houve uma maior conexão entre os autores em 2012, um total de 26\%, ou seja, 14 ocorrências. No entanto, essa conexão se estabilizou nos períodos subsequentes, com variação entre nove, 17\%, e dez ligações, 19\%. Tais resultados demonstram a importância desses encontros para estreitar relações e estimular laços, bem como para mapear as interrelações dos agentes na produção do conhecimento (Figura 2).

As medidas descritivas das redes são um dos elementos básicos na análise que envolve as interrelações sociais de indivíduos conectados em rede. Essas medidas resumem os aspectos estruturais das relações em vários níveis de análise, começando com uma unidade (apenas um nó), progredindo por meio de duplas (díades), trios (tríades) e vários grupos. Tais conceitos ajudam a examinar importantes características substantivas de nós, de grupos distintos e da rede (MAOZ, 2011).

Figura 2: Evolução na produção Científica da Revista ADMpg Gestão Estratégica

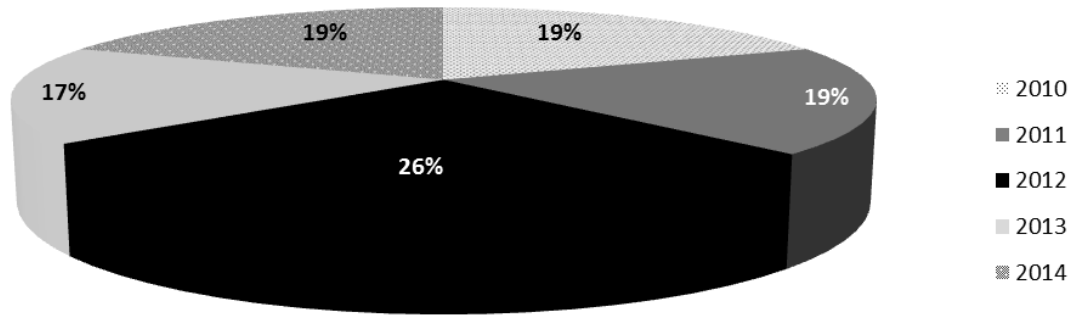

Fonte: Dados da pesquisa (2015)

Página 163 Caderno de Ciências Sociais Aplicadas, Vitória da Conquista/BA, vol. 15, n 25, ano 15, p. 155-177, jan/jun 2018. 


\section{- Cadernos de Ciénclas SOCIAIS APLICADAS}

\subsection{Grau de centralidade de Freeman, grau de intermediação e sociograma}

Visando avaliar a evolução anual da quantidade de ligações entre autores e suas respectivas instituições, na Tabela 1 são apresentadas as estatísticas descritivas, por meio das representações percentuais das medidas de dispersão: para média, soma, desvio-padrão, variância, grau máximo e mínimo das ligações nas redes de cooperação. Os resultados dos testes indicaram o grau de entrada e de saída de todos os nós presentes na rede. Dessa forma, ao interpretar esses resultados, concluímos que, as ligações entre atores e instituições ocorreram em 18\% de uma amostra de 53 artigos publicados em parceria, o que representa um total de 173 autores que interagiram em díades, tríades e em grupos com mais de três autores.

Com o intuito de identificar a quantidade de artigos publicados em parceria por atores pertencentes a uma mesma instituição, verificou-se a produção para todo o período. Os resultados apontaram um total de 91 artigos sem conexão. Desse total, a maior concentração ocorreu entre 2010 e 2011, com 23 e 20 artigos, respectivamente. Em 2012, foram 15 artigos; em 2013, um total de 17, e em 2014, 16 publicações.

Tabela 1: Análise descritiva para o grau de centralidade.

\begin{tabular}{lll}
\hline ESTATÍSTICAS & GRAU DE SAÍDA & GRAU DE ENTRADA \\
DESCRITIVAS & NORMALIZADO \% & NORMALIZADO \% \\
\hline Média & 1.807 & 1.807 \\
Desvio-padrão & 0.724 & 0.763 \\
Variância & 0.525 & 0.525 \\
Mínimo & 0.000 & 0.000 \\
Máximo & 4.624 & 4.624 \\
Soma & 314.451 & 314.451 \\
Total obs & 173.000 & 173.000 \\
\hline
\end{tabular}

Fonte: Dados da pesquisa (2015).

Nesta seção são apresentados os atores centrais na estrutura da rede, com destaque para os papeis de cada representante do grupo, os percentuais de centralidade e intermediações dos pesquisadores das instituições em estudo. A centralidade de determinado ator implica identificar a posição ocupada em relação às trocas e a comunicação existente na rede. Essa posição de centralidade e comunicação nas redes sociais traz a ideia de poder para os atores centrais, ou seja, quanto mais central é um ator mais bem posicionado ele está em relação às trocas e às ligações com outros autores, o que aumenta seu poder na rede (BURT, 1992; MARTELETO, 2001).

Página 164 Caderno de Ciências Sociais Aplicadas, Vitória da Conquista/BA, vol. 15, n 25, ano 15, p. 155-177, jan/jun 2018. 
Alguns aspectos ligados a redes sociais evidenciam traços que as caracterizam da seguinte forma: a análise motivada por uma intuição estrutural cuja base são os laços que ligam os atores sociais; os aspectos fundamentados em dados empíricos sistemáticos; e a análise fortemente amparada em imagens gráficas e modelos matemáticos e/ou computacionais empregados na análise das relações entre os atores de uma rede social (FREEMAN, 2004).

A importância de cada ator em uma rede pode ser identificada por meio de métricas de centralidade (De Souza, Barbastefano e De Lima, 2012). O apêndice 1 traz a relação completa com informações de medidas de centralidade "Tabela 2" e intermediação "Tabela 3" dos atores. No que tange à rede de cooperação entre as coautorias, neste estudo, optamos por mostrar apenas o sociograma dos doze autores mais bem posicionados em grau de centralidade e intermediação. Ressaltase que apenas ocorreram ligações entre esses indivíduos e, dessa forma, o quadro de ligações aparece de forma reduzida, ou seja, considerando 0,000 para todo o restante da amostra.

O objetivo da análise foi evidenciar o grau de entrada e de saída correspondente ao fluxo e direção nas ligações entre os agentes. Silva et al. (2006) destacam que as análises de redes são feitas por meio de matrizes binárias, com informações que demonstram apenas a existência de laços de coautorias e matrizes valoradas com informações sobre o número de colaborações entre os atores. As conexões entre os autores dos artigos com o maior grau de ligações estão dispostas na Figura 3. Para Andrade e Rego (2015), coautoria implica uma relação temporal e acadêmica, em que há o compartilhamento de ideias e recursos, diferentemente das citações, que, em alguns casos, ocorrem sem o conhecimento dos autores.

Página 165 Caderno de Ciências Sociais Aplicadas, Vitória da Conquista/BA, vol. 15, n 25, ano 15, p. 155-177, jan/jun 2018. 


\section{- Cadernos de CIIENCIAS SOCIAIS APLICADAS}

Figura 3: Estrutura das redes de cooperação entre os autores.

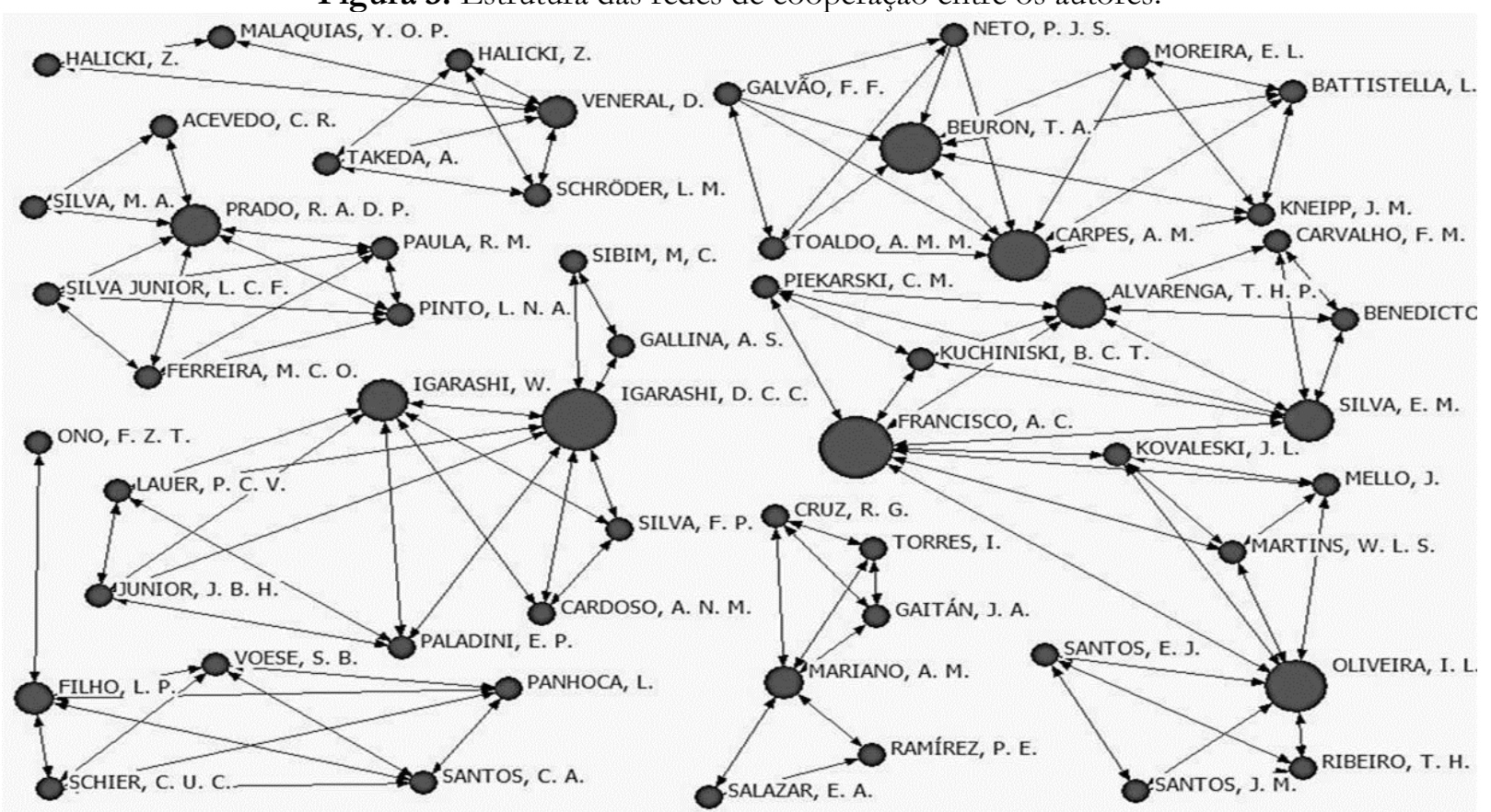

Fonte: Dados da pesquisa (2015).

Os resultados levaram a concluir que IGARASHI, D. C. C. e FRANCISCO, A. C possuem o maior grau de centralidade, $4.6 \%$. Com isso, são os atores mais bem posicionados, considerando a difusão, o fluxo e o poder de aproximar e intermediar a comunicação entre os agentes da rede. $\mathrm{Na}$ sequência, estão OLIVEIRA, I. L.; BEURON, T. A., CARPES; A. M., com 4.0\% de intermediação, seguidos por IGARASHI, W.; ALVARENGA, T. H. P.; SILVA, E. M.; PRADO, R. A. D. P., com 3.5\% e VENERAL, D.; FILHO, L. P., MARIANO, A. M., com 2.9\%. A análise completa de ligações apontou a formação de díades (5 nós), tríades (15 nós), ficando o restante com mais de três coautores.

Pesquisadores com maior nível de centralidade tendem a atingir maiores níveis de produtividade. Entretanto, essa tendência se aplica para os pesquisadores que assumem um papel de “intermediação”, tendo em vista o controle no fluxo de informação (ANDRADE e REGO, 2015).

A seguir são apresentados os escores para medidas de centralidade de Freeman e intermediação das instituições de ensino mais prolíficas em relação a coautorias. Nesse cenário, podemos destacar na Tabela 4 os principais autores centrais dessa rede e suas respectivas instituições: Universidade Tecnológica Federal do Paraná (UTFPR), com 10\% de centralidade. Em segundo lugar, a Universidade Federal do Paraná (UFPR), com 8\%, seguida da Universidade Estadual do Oeste do Paraná (UNIOESTE), com 7\%.

Página 166 Caderno de Ciências Sociais Aplicadas, Vitória da Conquista/BA, vol. 15, n 25, ano 15, p. 155-177, jan/jun 2018. 
Ao analisar as medidas do grau de intermediação das instituições, constatamos uma mudança de posição entre a UFPR e a UFSC. As instituições UTFPR, UNIOESTE e UFSC obtiveram, respectivamente, os seguintes graus de intermediação: 3.905, 2.989 e 1.491. O posicionamento de determinado ator em uma rede caracteriza a capacidade de criar laços e ligações com os demais membros. Inferimos que, no processo intermediações, esse controle pode ser interpretado como a possibilidade que um nó tem para intervir nas comunicações entre os agentes dessa rede. Tais implicações levam em consideração a importância do ator na intermediação e a possibilidade de um nó estreitar laços com os outros pares de nós.

Tabela 4: Medidas - Grau de centralidade de Freeman e intermediação das instituições.

\begin{tabular}{|c|c|c|c|c|c|c|c|c|}
\hline INSTITUIÇÃO & G.C. & G.I. & INSTITUIÇÃO & G.C. & G.I. & INSTITUIÇÃO & G.C. & G.I. \\
\hline UTFPR & 0.101 & 3.905 & UEL & 0.020 & 0.021 & UNINORTE & 0.010 & 0.000 \\
\hline UFPR & 0.081 & 0.618 & FAESI & 0.020 & 0.000 & FAMAM & 0.010 & 0.000 \\
\hline UNIOESTE & 0.071 & 2.989 & SECAL & 0.020 & 0.000 & UFRB & 0.010 & 0.000 \\
\hline UFU & 0.051 & 0.186 & USP & 0.020 & 0.000 & KRAEMER & 0.010 & 0.000 \\
\hline UFSC & 0.051 & 1.491 & IFMS & 0.020 & 0.000 & SENAI & 0.010 & 0.000 \\
\hline UEM & 0.040 & 0.910 & F.ANHANG. & 0.020 & 0.000 & U. C.D. NORTE & 0.010 & 0.000 \\
\hline UNICENTRO & 0.040 & 0.807 & UNIDERP & 0.010 & 0.000 & UNB & 0.010 & 0.000 \\
\hline UNIP & 0.030 & 0.082 & U. SALAMAN & 0.010 & 0.000 & LATEC - UFF & 0.000 & 0.000 \\
\hline UFLA & 0.030 & 0.989 & UTAD & 0.010 & 0.000 & FUNCESI & 0.000 & 0.000 \\
\hline UNESP & 0.030 & 0.989 & U. S.ESPAÑA & 0.010 & 0.000 & ITE & 0.000 & 0.000 \\
\hline UFSM & 0.030 & 0.625 & U. TUIUTI/PR & 0.010 & 0.000 & UDESC & 0.000 & 0.000 \\
\hline UNIPAMPA & 0.030 & 0.368 & UNEMAT & 0.010 & 0.000 & U. D. BÍO-BÍO & 0.000 & 0.000 \\
\hline IFSP/FATEC & 0.030 & 0.000 & UFV & 0.010 & 0.000 & U. A. MÉXICO & 0.000 & 0.000 \\
\hline UNIVÁS & 0.030 & 0.000 & FATEC-BS & 0.010 & 0.031 & UFF & 0.000 & 0.000 \\
\hline UNITAU & 0.030 & 0.000 & FEI & 0.010 & 0.000 & IFES & 0.000 & 0.000 \\
\hline UNIVALI & 0.030 & 0.000 & FAFIT & 0.010 & 0.000 & UFC & 0.000 & 0.000 \\
\hline UNINOVE & 0.030 & 0.103 & FACINTER & 0.010 & 0.000 & UBM & 0.000 & 0.000 \\
\hline CESREAL & 0.030 & 0.000 & UNIME & 0.010 & 0.000 & UVA & 0.000 & 0.000 \\
\hline UNOESC & 0.030 & 0.000 & IPCA & 0.010 & 0.000 & U. D.M.PORT & 0.000 & 0.000 \\
\hline IFPR & 0.030 & 0.000 & UPET & 0.010 & 0.000 & SETREM & 0.000 & 0.000 \\
\hline UFPE & 0.020 & 0.989 & FACE & 0.010 & 0.000 & UNIFRA & 0.000 & 0.000 \\
\hline UFBA & 0.020 & 0.268 & UFPA & 0.010 & 0.000 & FAE & 0.000 & 0.000 \\
\hline UFS & 0.020 & 0.000 & UNAMA & 0.010 & 0.000 & US.CAMIL. & 0.000 & 0.000 \\
\hline UEPG & 0.020 & 0.974 & UFCG & 0.010 & 0.000 & UEMS & 0.000 & 0.000 \\
\hline UNAM & 0.020 & 0.000 & UNESC & 0.010 & 0.000 & UESC & 0.000 & 0.000 \\
\hline UNIJUI & 0.020 & 0.000 & PUC-PR & 0.010 & 0.000 & UNINTER & 0.000 & 0.000 \\
\hline FAC. SPEI & 0.020 & 0.320 & M. D.BRASIL & 0.010 & 0.000 & IFC & 0.000 & 0.000 \\
\hline UFMT & 0.020 & 0.052 & FGV & 0.010 & 0.000 & UNIÃO & 0.000 & 0.000 \\
\hline $\mathrm{UCB}$ & 0.020 & 0.000 & FIP/Magsul & 0.010 & 0.000 & UNISINOS & 0.000 & 0.000 \\
\hline UNIGRANRIO & 0.020 & 0.000 & FAHOR & 0.010 & 0.000 & $\begin{array}{l}\text { UNOCHAPEC } \\
\text { O }\end{array}$ & 0.000 & 0.000 \\
\hline UFGD & 0.020 & 0.021 & UNIJUÍ & 0.010 & 0.000 & UFPB & 0.000 & 0.000 \\
\hline CESUPA & 0.020 & 0.000 & $\begin{array}{l}\text { FESG/FAFIC } \\
\mathrm{H}\end{array}$ & 0.010 & 0.000 & UNIVILLE & 0.000 & 0.000 \\
\hline IFAM & 0.020 & 0.000 & U. EVANG. & 0.010 & 0.000 & & & \\
\hline SEPLAN & 0.020 & 0.000 & FMU & 0.010 & 0.000 & & & \\
\hline
\end{tabular}

Nota: G.C - Grau de centralidade, G.I - Grau de intermediação.

Página 167 Caderno de Ciências Sociais Aplicadas, Vitória da Conquista/BA, vol. 15, n 25, ano 15, p. 155-177, jan/jun 2018. 
Fonte: Dados da pesquisa (2015).

$\mathrm{Na}$ Figura 4 é possível visualizar as redes sociais observadas na produção de artigos científicos publicados no periódico. O resumo dos dados evidenciados no sociograma demonstra a posição central da formação da rede. Entre outras instituições, o destaque é para a UTFPR, em termos de centralidade e grau de intermediação. A UFPR, a UNIOESTE, a UFU e a UFSC também ocupam papel de destaque na inter-relação dos autores, representada de acordo com a posição de cada ator em relação aos demais.

Figura 4: Estrutura das redes de cooperação entre as Instituições.

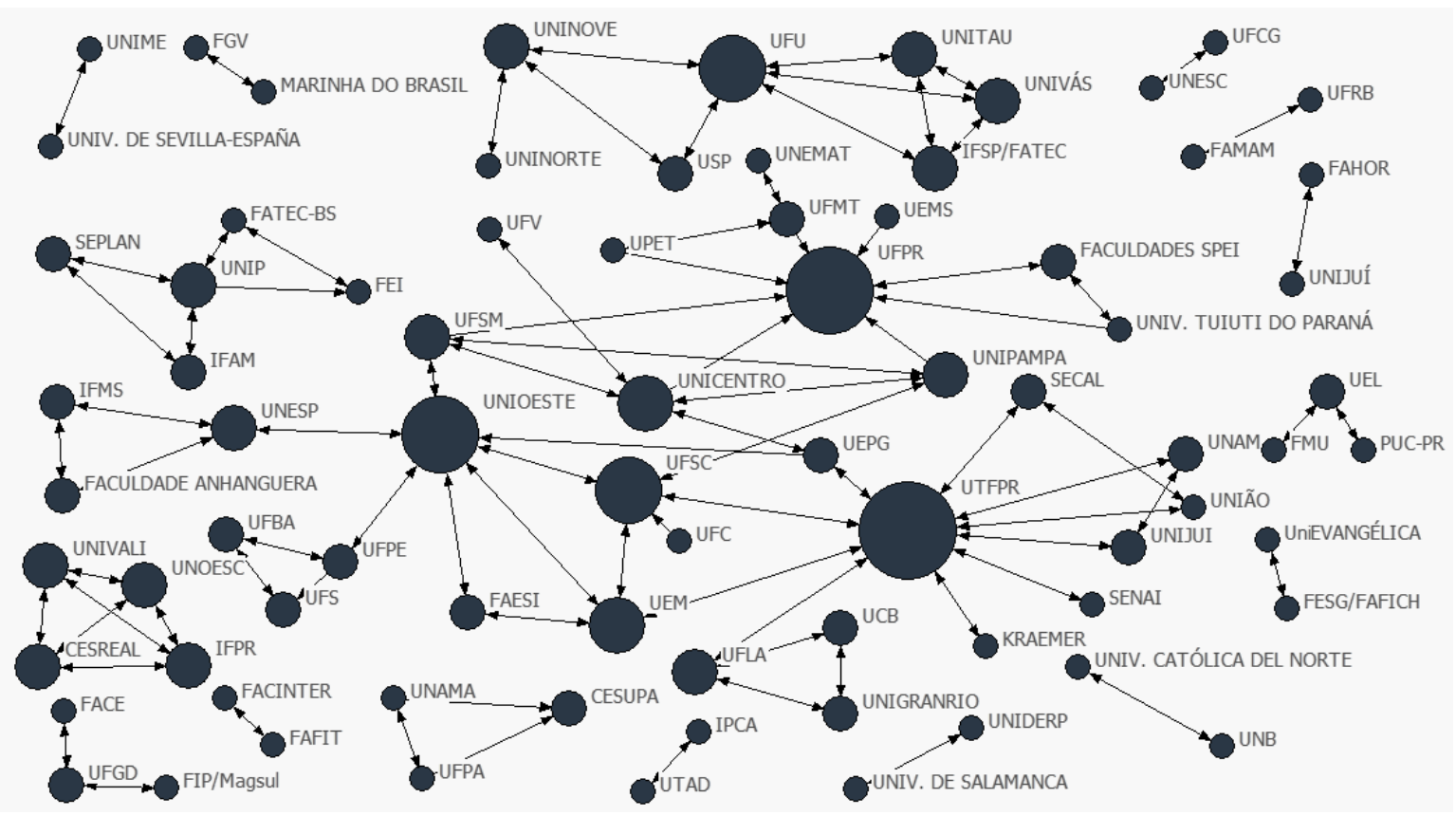

Fonte: Dados da pesquisa (2015).

A análise de redes sociais permite entender a dinâmica das relações entre os autores, identificar a troca, os fluxos de informação, os mecanismos e agentes de poder, bem como analisar as ligações em ambientes complexos (BRANDÃO, PARREIRAS e SILVA, 2007).

Segundo Silva et al. (2006), o número de pesquisas sobre redes sociais vem crescendo constantemente nas várias áreas do conhecimento. O principal interesse dos estudiosos é compreender o seu impacto na vida social. Isso tem originado inúmeras metodologias de análise, as quais têm por base as relações sociais dos indivíduos. No Brasil, alguns estudos têm sido realizados para verificar essas relações no âmbito da produção científica. Entre esses, destacam-se: Machado (2007), Maia e Caregnato (2008), Walter et al. (2009) e Nascimento e Beuren (2011).

Página 168 Caderno de Ciências Sociais Aplicadas, Vitória da Conquista/BA, vol. 15, n² 25, ano 15, p. 155-177, jan/jun 2018. 
Utilizando-se de metodologia qualitativa e descritiva, Machado (2007) realizou uma análise com ênfase em estudos ocorridos entre 1990 e 2005, nas áreas de Biblioteconomia e de Ciência da Informação. Segundo o autor, os resultados demonstraram uma assimetria que varia entre um e seis artigos por ano, com o predomínio de um único autor. Além disso, constatou-se que a Revista Ciência da informação foi a mais prolífica no período em questão.

Maia e Caregnato (2008) analisaram as redes de colaboração científica entre os docentes do programa de pós-graduação em epidemiologia da Universidade Federal de Pelotas. Conforme revelam os achados da pesquisa, o número de artigos com autoria compartilhada foi maior do que as publicações individuais, não sendo possível detectar a relação entre o aumento da produtividade e o número de colaboradores. Walter et al. (2009) identificaram os atores que mais se destacaram em termos de produção científica sobre ensino e pesquisa contábil no Brasil. Os resultados apontam uma evolução nessa área no que se refere ao número de publicações e à densidade das redes de cooperação.

O estudo de Nascimento e Beuren (2011) teve o objetivo de identificar a formação de redes sociais na produção científica definitiva dos programas de pós-graduação de ciências contábeis do Brasil, no triênio 2007-2009. Os resultados mostraram que a evolução da produção científica definitiva, em termos percentuais, foi maior nos programas com o conceito 3. Evidenciaram, além disso, fracas ligações, dispersão e baixa densidade nas estratificações do Qualis CAPES.

O mapeamento no acervo das produções científicas na área da administração já foi objeto de várias pesquisas. Estudos sobre redes sociais e bibliometria similares a este, analisaram a produção acadêmica em revistas nacionais, como: Bulgacov e Verdu, 2001; Teixeira, Iwamoto e Medeiros, 2013; Ribeiro, 2015; Favaretto e Francisco, 2017.

Bulgacov e Verdu (2001) observaram as relações em rede existentes entre os pesquisadores que participaram do encontro anual da ANPAD. Segundo os autores, houve pouca participação dos pesquisadores em redes, apesar do uso sistemático de novas ferramentas tecnológicas.

Teixeira, Iwamoto e Medeiros (2013) verificaram a produção científica nos principais periódicos nacionais, classificados no sistema qualis "estratos B1 e A2", na área de Administração no Brasil. Os resultados desse estudo indicam que artigos apresentam categorias que nem sempre contemplam a técnica bibliométrica. Nessa concepção, os autores sugerem uma melhor adequação do método aplicado a novas pesquisas.

Ribeiro (2015) explorou a produção acadêmica científica da Revista de Administração Contemporânea (RAC), no período entre 1997 e 2011. Os atores mais prolíficos foram: Machado-da-

Página 169 Caderno de Ciências Sociais Aplicadas, Vitória da Conquista/BA, vol. 15, n 25, ano 15, p. 155-177, jan/jun 2018. 
Silva, Carrieri, Vasconcelos e Rezende, com destaque para as seguintes Instituições de Ensino Superior (IES): USP, FGV (SP), UFRGS, UnB, UFPR, UFMG e UFRJ, as quais contribuíram para ampliar os estudos de redes sociais na área de Administração do Brasil.

Favaretto e Francisco (2017) realizaram um estudo das cinco décadas (entre 1961 e 2016) do acervo da Revista de Administração de Empresas (RAE), com a utilização de técnicas de bibliometria, text mining, rede social e geoanálise. As evidências mostram que, para o período entre 2013 e 2016, a colaboração é cada vez mais valorizada entres os autores interligados na rede de coautorias, em periódicos científicos de Administração.

Os estudos científicos publicados na Revista ADMpg Gestão e Estratégia constituem um respeitável veículo de comunicação, com um papel importante no processo de troca de informações e disseminação do conhecimento, na medida em que a difusão de ideias na formação de redes sociais propiciam a integração de novos membros a futuros projetos de pesquisas.

\section{Considerações finais}

A troca de conhecimento na produção científica exerce impacto na vida social, possibilita a integração de atores, o estreitamento de laços e estimula novas pesquisas. O objetivo deste estudo foi verificar a formação de redes sociais na produção científica da revista ADMpg Gestão Estratégica, bem como a constituição de laços de coautorias na estrutura dessas redes.

Os termos presentes nos artigos com o maior número de ocorrências, segmentados em títulos, resumos e palavras-chave foram: pesquisa, empresas, estudo, objetivo e sustentabilidade. O período de maior interação dos coautores foi o ano de 2012. Nos anos subsequentes, e também nos anteriores, o quadro estabilizou-se, variando entre nove e dez ligações entre os autores. Os resultados demonstram a importância desses encontros para estreitar relações e estimular a formação de laços, permitindo a interrelação dos agentes na produção do conhecimento.

O estudo indicou a formação de redes entre grupos dispersos. Mostrou, além disso, as instituições com maior grau de centralidade: a UTFPR, a UFPR, seguida da UNIOESTE, as quais ocupam papel de destaque na inter-relação e intermediação dos autores, na criação de laços com outras instituições para a cooperação entre os participantes da rede.

Os principais autores em grau de centralidade e intermediações dessa rede são IGARASHI, D. C. C. FRANCISCO, A. C. e OLIVEIRA, I. L. Os resultados levam a concluir que a formação de rede e

Página 170 Caderno de Ciências Sociais Aplicadas, Vitória da Conquista/BA, vol. 15, n 25, ano 15, p. 155-177, jan/jun 2018. 
a cooperação entre os atores expõem fracas ligações e conexões esparsas, mediante o restrito número de ligações e grupos isolados. Em relação ao grau de centralidade, as três IES do estado do Paraná, região Sul, apresentam maior possibilidade de um ator exercer controle sobre os demais dependentes para realizar ligações. Isso ocorre possivelmente pela localização geográfica, uma vez que o Congresso Internacional de ADM premia os melhores artigos do evento e os enviam ao periódico. A produção científica e o relacionamento entre os agentes mantêm-se ainda de forma modesta, levando em conta que não houve um crescimento significativo no número de coautorias entre as instituições participantes.

A principal limitação deste estudo refere-se aos aspectos metodológicos, dado que não há uma análise do discurso, o que demandaria maior tempo de investigação, tendo em vista o tamanho da amostra. Constituem sugestões para pesquisas futuras: (a) aplicar o mesmo estudo com a ampliação do período de análise; (b) considerar na amostra análises comparativas por área do conhecimento.

\section{Referências}

ALDRICH, H. E. Organizations and environments. New Jersey: Prentice-Hall, 1979.

ANDRADE, R. L.; REGO, L. C. A Influência da rede de coautoria no nível das bolsas de produtividade da área de engenharia de produção. In: IV BRAZILIAN WORKSHOP ON SOCLAL NETWORK ANALYSIS AND MINING (BraSNAM), 2015, Recife. Anais... Recife, 2015.

Conhecendo a rede de coautoria dos bolsistas de produtividade em pesquisa da área de engenharia de produção e a sua influência no nível de produtividade. In: XLVII SIMPÓSIO BRASILEIRO DE PESQUISA OPERACIONAL - SBPO, 2015, Recife. Anais... Recife, 2015.

APOSTOLATO, I. A. An overview of Software Applications for social network analysis, International Review of Social Research, v. 3, n. 3, p. 71-77, 2013.

BASTOS, M. T.; ZAGO, G. S.; RECUERO, R. A endogamia da comunicação: redes de colaboração na CSA 1. Revista FAMECOS (Online), v. 23, p. 1-22, 2016.

BRANDÃO, W. C.; PARREIRAS, F. S.; SILVA, A. B. O. Redes em ciência da informação: evidências comportamentais dos pesquisadores e tendências evolutivas das redes de co-autoria. Informação \& Informação. v. 12, 2007.

BRITO, J. Cooperação Interindustrial e Redes de Empresas. In: KUPFER, D.; HASENCLEVER, L. Economia Industrial: fundamentos teóricos e práticos no Brasil. Rio de Janeiro: Campus, 2002.

BULGACOV, S; VERDU, F. Redes de pesquisadores da área de administração: um estudo exploratório. Revista de Administração Contemporânea - RAC, Edição Especial, p. 163-182, 2001.

Página 171 Caderno de Ciências Sociais Aplicadas, Vitória da Conquista/BA, vol. 15, n 25, ano 15, p. 155-177, jan/jun 2018. 
BURT, R. S. Structural holes: the social structure of competition. Cambridge, MA: Harvard University Press, 1992.

CARRINGTON, P.J.; SCOTT, J.; WASSERMAN, S. Models and methods in social network analysis. Cambridge University Press, Cambridge, p. 270-316, 2005.

COOPER, D. R.; SCHINDLER, P. S. Métodos de pesquisa em administração. (10ª ed.). Porto Alegre: Bookman, 2011.

CORRELL M.; ALEXANDER, E. C.; GLEICHER, M. Quantity estimation in visualizations of tagged text. In: Proceedings of the SIGCHI conference on buman factors in computing systems. New York: ACM. p. 26972706, 2013.

DE SOUZA, C.G., BARBASTEFANO, R.G.; DE LIMA, L.S. Chemistry colaboration networks in Brazil: A coauthorship study in quimica nova articles. Quimica Nova, v.15, n.4, p. 671-676, 2012.

EVERETT', M. G.; BORGATTI, S. P. Models and methods in social network analysis extending centrality. In: CARRINGTON, P., SCOT'T, J. WASSERMAN, S. (organizadores). New York: Cambridge Press, 2005.

FAVARETTO, J. E.; FRANCISCO, E. R. Exploração do acervo da RAE- Revista de Administração de Empresas (1961 a 2016) à luz da bibliometria, text mining, rede social e geoanálise. Revista de Administração de Empresas - RAE, v. 57, n. 4, p. 365-390, 2017.

FLICK, U. Introdução à pesquisa qualitativa. Trad. Joice Elias Costa. 3. ed. Porto Alegre: Artmed, 2004.

FREEMAN, L. C. Some antecedents of social network analysis. Connections, v. 19, n.1, p. 39-42, 1996.

2004.

. C. The development of social network analysis: a study in the sociology of science. Vancouver: Empirical,

GIL, A. C. Métodos e técnicas de pesquisa social. (6. Ed). São Paulo: Atlas, 2008.

GUIMARÃES, T. A.; GOMES, A. O.; ODELIUS, C. C.; ZANCAN, C.; CORRADI, A. A. A rede de programas de pós-graduação em administração no Brasil: análise de relações acadêmicas e atributos de programas. Revista de Administração Contemporânea, v. 13 n. 4, p. 564-582, 2009.

HANNEMAN, R., RIDLLE, M. Introduction to social network methods. Riverside: University of California, 2005.

LEMOS, L. M. P. Nuvem de tags como ferramenta de análise de conteúdo: uma experiência com as cenas estendidas da telenovela Passione na internet. Lumina (UFJF. Online), v. 10, p. 1-18, 2016.

MACHADO, D. Q.; IPIRANGA, A. S. R.; MATOS, F. R. N. Das redes sociais às redes interorganizacionais: a evolução das abordagens cooperativas na sociedade em rede. Revista Brasileira de Administração Cientifica, Aquidabã. v. 4, n. 1, p. 79- 103, 2013.

Página 172 Caderno de Ciências Sociais Aplicadas, Vitória da Conquista/BA, vol. 15, n 25, ano 15, p. 155-177, jan/jun 2018. 
MACHADO, R. N. Análise cientométrica dos estudos bibliométricos publicados em periódicos da área de biblioteconomia e ciência da informação (1990-2005). Perspectivas em ciência da informação, v.12, n.3, p. 2-20, 2007.

MAIA, M. F. CAREGNATO, S. E. Co-Authorship as an indicator of scientific Collaboration Network. Perspectivas em Ciência da Informação, Vol. 13, No. 2,pp. 18 31, 2008.

MALAGOlli, G. A.; PAUlillo, L. F. O. Mobilização Política e Rede de Interesses na Produção Calçadista de Jaú. Gestão \& Produção (UFSCAR. Impresso), v. 20, p. 927-938, 2013.

MAOZ. Z. Networks of nations the evolution, structure, and impact of international networks, New York: Cambridge University Press, p. 1816-2001, 2011.

MARTELETO, R. M. Análise de redes sociais: aplicação nos estudos de transferência da informação. Ciência da Informação, v. 30, n. 1, p. 71-81, 2001.

MARTES, A. C. B.; BULGACOV, S.; NASCIMENTO, M. R.; GONÇALVES, S. A.; AUGUSTO, P. M. Fórum - Redes sociais e interorganizacionais. Revista de Administração de Empresas, v. 46, n. 3, p. 10$15,2006$.

MCNAUGHT, C.; LAM, P. Using wordle as a supplementary research tool. The Qualitative Report, v. 15, n. 3, p. 630-643, 2010.

MIZRUCHI, M. Análise de redes sociais: avanços recentes e controvérsias atuais. Revista de Administração de Empresas, v. 46, n. 3, p. 73-85, 2006.

MOREIRA, S. V. Análise documental como método e como técnica. In: DUARTE, J., BARROS, A. (Orgs.). Métodos e técnicas de pesquisa em comunicaşão. São Paulo: Atlas, p. 269-279, 2005.

NASCIMENTO, S.; BEUREN, I. M. Redes sociais na produção cientifica dos programas de pósgraduação de Ciências Contábeis do Brasil. RAC, Curitiba, v. 15, n. 1, p. 47-66, 2011.

NELSON, R. O uso da Análise de Redes Sócias no estudo das estruturas organizacionais. Revista de Administração de Empresas, v. 24, n. 4, p. 150-157, 1984.

PRYKE, S. Social network analysis in construction. Chichester: Wiley-Blackwell, 2012.

RIBEIRO, H. C. M. Quinze Anos de Estudo da Revista de Administração Contemporânea sob a Ótica da Bibliometria e da Rede Social. Perspectivas em Gestão \& Conhecimento, v. 5 (Número Especial), p. 86108, 2015.

RIVADENEIRA, A.W.; GRUEN, D. M.; MULLER, M. J. MILLEN, D. R. Getting our head in the clouds: toward evaluation studies of tagclouds. In: PROCEEDINGS OF THE SIGCHI CONFERENCE ON HUMAN FACTORS IN COMPUTING SYSTEMS, p. 995-998, 2007.

SCOTT, J. Social Network, Analysis: a bandbook. 2 ed., London: Sage Publications, 2000.

Página 173 Caderno de Ciências Sociais Aplicadas, Vitória da Conquista/BA, vol. 15, n 25, ano 15, p. 155-177, jan/jun 2018. 
SILVA, A. B. O. E. ; MATHEUS, R. F.; PARREIRAS, F. S.; PARREIRAS, T. A. S. Estudo da rede de co-autoria e da interdisciplinaridade na produção científica com base nos métodos de análise de redes sociais: avaliação do caso do Programa de Pós-Gradução em Ciência da Informação - PPGCI / UFMG. Enc. Bibli: R. Eletr. Bibliotecon. Ci. Inf., Florianópolis, n. esp., $1^{\circ}$ sem. 2006.

TEIXEIRA, M. L. M.; IWAMOTO, H. M.; MEDEIROS, A. L. Estudos bibliométricos (?) Em administração: discutindo a transposição de finalidade. Administração: Ensino e Pesquisa, Rio de Janeiro v. 14, n. 3, p. 423-452, 2013.

TOMAÉL, M. I.; ALCARÁ, A.R.; CHIARA, I. G. D. Das redes sociais à inovação. Ciência da Informação. Brasilia, v. 34, n. 2, p. 93-104, 2005.

VIANA, F. L. E.; BALDI, M. Contribuições da teoria de redes à gestão da cadeia de suprimento. In: XXXII Encontro da ANPAD, 2008, Rio de Janeiro. XXXII ENANPAD. Rio de Janeiro: ANPAD, 2008.

WALTER, S. A.; CRUZ, A. P. C.; ESPEJO, M. M. S. B.; GASSNER, F. P. Uma análise da evolução do campo de ensino e pesquisa em contabilidade sob a perspectiva de redes. In: CONGRESSO USP DE CONTROLADORIA E CONTABILIDADE, 9., 2009, São Paulo. Anais... São Paulo: FEA/USP, 2009.

WASSERMAN, S.; FAUST, K. Social network analysis: methods and applications. Cambridge: Cambridge University Press, 1994.

WEIWEI CUI, Y. W.; SHIXIA LIU, F. W.; MICHELLE, X. Z.; HUAMIN, QU. Contextpreserving, dynamic word cloud visualization. IEEE Computer Graphics and Applications, In: IEEE Pacific Visualisation Symposium. Taiwan. v. 30, n. 6, p. 42-53, 2010.

WILLIAMSON, O. E. Markets and Hierarchies: analysis and antitrust implications. NewYork: Free Press, 1975.

ZHANG, M. Social Network Analysis: History, Concepts, and Research. In: Handbook of Social Network Technologies and Applications. Editor Borko Furht. Springer, 2010.

Página 174 Caderno de Ciências Sociais Aplicadas, Vitória da Conquista/BA, vol. 15, n 25, ano 15, p. 155-177, jan/jun 2018. 


\section{Apêndice - 1}

Tabela 2: Medidas - Grau de centralidade de Freeman de coautorias.

\begin{tabular}{|c|c|c|c|}
\hline AUTOR & G.C. $(\%)$ & AUTOR & G.C. $(\%)$ \\
\hline IGARASHI, D. C. C. & 0.046 & CARDOSO, A. N. M. & 0.017 \\
\hline FRANCISCO, A. C. & 0.046 & SILVA, F. P. & 0.017 \\
\hline OLIVEIRA, I. L. & 0.040 & TORRES, I. & 0.017 \\
\hline BEURON, T. A. & 0.040 & SILVA, N. P. & 0.017 \\
\hline CARPES, A. M. & 0.040 & SANTI, R. C. & 0.017 \\
\hline IGARASHI, W. & 0.035 & DEGRAF, D. M. & 0.017 \\
\hline ALVARENGA, T. H. P. & 0.035 & SILVA, M. C. G. & 0.017 \\
\hline SILVA, E. M. & 0.035 & WEISE, A. D. & 0.017 \\
\hline PRADO, R. A. D. P. & 0.035 & BARCHET, I. & 0.017 \\
\hline VENERAL, D. & 0.029 & SILUK, J. C. M. & 0.017 \\
\hline FILHO, L. P. & 0.029 & SCHRIPPE, P. & 0.017 \\
\hline MARIANO, A. M. & 0.029 & CARVALHO, F. M. & 0.017 \\
\hline PALADINI, E. P. & 0.023 & BENEDICTO, G. C. & 0.017 \\
\hline RODRIGUES, J. F. & 0.023 & CARDOSO, P. C. S. & 0.017 \\
\hline LIMA, I. A. & 0.023 & CASAS, J. L. S. & 0.017 \\
\hline TEIXEIRA, E. B. & 0.023 & SILVA, D. O. & 0.017 \\
\hline PILATTI, L. A. & 0.023 & SANTOS, E. A. F & 0.017 \\
\hline PONTES, C. K. & 0.023 & SANTOS, J. M. & 0.017 \\
\hline DANGO, L. A. & 0.023 & SANTOS, E. J. & 0.017 \\
\hline VIEIRA, R. A. & 0.023 & RIBEIRO, T. H. & 0.017 \\
\hline ARRUDA, L. L. & 0.023 & TUBINO, D. F. & 0.012 \\
\hline FREITAG, V. C. & 0.023 & SILVA, P.R. & 0.012 \\
\hline NETO, M. S. N. & 0.023 & DEBOÇÃ, L. P. & 0.012 \\
\hline OLIVEIRA, H. H. & 0.023 & SILVA, A.J. & 0.012 \\
\hline BISAGGIO, R. S. & 0.023 & JUCHEM, D. M. & 0.012 \\
\hline LIMA, J. L. A. & 0.023 & ROSSATI, W. R. & 0.012 \\
\hline SACOMANO, J. B. & 0.023 & GOMES, N. B. & 0.012 \\
\hline MELLO, J. & 0.023 & MORAES, S. C. S. & 0.012 \\
\hline MARTINS, W. L. S. & 0.023 & NETO, P. L. O. & 0.012 \\
\hline KOVALESKI, J. L. & 0.023 & NORONHA, M. & 0.012 \\
\hline SANTOS, C. A. & 0.023 & ALEIXO, D. O. & 0.012 \\
\hline BACCIN, S. S. & 0.023 & HOLANDA, L. M. C. & 0.012 \\
\hline MAIOLI, M. R. & 0.023 & CÂNDIDO, G. A. & 0.012 \\
\hline PALOSCHI, M. M. W. & 0.023 & SIBIM, M, C. & 0.012 \\
\hline SILVA, G. E. & 0.023 & SEREIA, V. J. & 0.012 \\
\hline VIOLIN, F. L. & 0.023 & STAL, E. & 0.012 \\
\hline RIBEIRO, R. M. & 0.023 & CAMARA, M. R. G. & 0.012 \\
\hline BUSCIOLI, R. R. & 0.023 & GAZEL, W. F. & 0.012 \\
\hline VIOLIN, A. L. & 0.023 & FEITOSA, W. G. & 0.012 \\
\hline VIOLIN, P. K. & 0.023 & SALLES, J. A. A. & 0.012 \\
\hline SCHIER, C. U. C. & 0.023 & SILVA, M. A. & 0.012 \\
\hline PANHOCA, L. & 0.023 & ACEVEDO, C. R. & 0.012 \\
\hline VOESE, S. B. & 0.023 & RAMÍREZ, P. E. & 0.012 \\
\hline SILVA, M. M. & 0.023 & FRANCO, C. & 0.012 \\
\hline CARVALHO, A. M. & 0.023 & BONJOUR, S. C. M. & 0.012 \\
\hline CORRÊEA, E. A. & 0.023 & PEREIRA, B. D. & 0.012 \\
\hline POTHER, S. G. & 0.023 & LEISMANN, E. L. & 0.012 \\
\hline BASTOS, T. N. & 0.023 & LAGIOIA, C. T. L. & 0.012 \\
\hline COSTA, V. L. & 0.023 & CARMONA, C. U. M. & 0.012 \\
\hline KOVALESKI, J. L. & 0.023 & NETO, J. F. R. & 0.012 \\
\hline
\end{tabular}

Página 175 Caderno de Ciências Sociais Aplicadas, Vitória da Conquista/BA, vol. 15, n 25, ano 15, p. 155-177, jan/jun 2018. 


\begin{tabular}{|c|c|c|c|}
\hline JUNIOR, P. P. A. & 0.023 & GALLEGO, P. A. M. & 0.012 \\
\hline COSTA, D. L. & 0.023 & SOUZA, C. C. & 0.012 \\
\hline MORSCHEL, E. L. & 0.023 & SILVA, N. A. S. & 0.012 \\
\hline KNEIPP, J. M. & 0.023 & FARIAS, T. A. & 0.012 \\
\hline MOREIRA, E. L. & 0.023 & SILVA, G. A. S. & 0.012 \\
\hline BATTISTELLA, L. F. & 0.023 & FERNANDES, P. & 0.012 \\
\hline JUNIOR, J. B. H. & 0.023 & VARAJÃO, J. & 0.012 \\
\hline LAUER, P. C. V. & 0.023 & CUNHA, M. & 0.012 \\
\hline EYNG, I. S. & 0.023 & MALAQUIAS, Y. O. P. & 0.012 \\
\hline KUCHINISKI, B. C. T. & 0.023 & HALICKI, Z. & 0.012 \\
\hline PIEKARSKI, C. M. & 0.023 & ELIAS, S. J. B. & 0.012 \\
\hline VEIGA, R. S. & 0.023 & OLIVEIRA, M. M. & 0.012 \\
\hline POLACINSKI, E. & 0.023 & PACAGNAN, M. N. & 0.012 \\
\hline SILVA, V. B. & 0.023 & GIMENEZ, F. A. P. & 0.012 \\
\hline TAUCHEN, J. & 0.023 & RUBO, M. & 0.012 \\
\hline PIRES, M. R. & 0.023 & HEIM, J. G. & 0.012 \\
\hline PINTO, L. N. A. & 0.023 & PEREIRA, W. C. & 0.012 \\
\hline FERREIRA, M. C. O & 0.023 & LEITE, A. S. & 0.012 \\
\hline PAULA, R. M. & 0.023 & GALVÃO, F. F. & 0.012 \\
\hline SILVA JUNIOR, L. C. F. & 0.023 & TOALDO, A. M. M. & 0.012 \\
\hline STADLER, A. & 0.023 & NETO, P. J. S. & 0.012 \\
\hline ABBAS, K. & 0.017 & TOLFO, C. & 0.012 \\
\hline LEONCINE, M. & 0.017 & MOMBACH, J. G. & 0.012 \\
\hline PONTES, J. & 0.017 & FERREIRA, M. G. G. & 0.012 \\
\hline QTA, E. M. & 0.017 & GALLINA, A. S. & 0.012 \\
\hline VIEIRA, C. B. & 0.017 & SALAZAR, E. A. & 0.012 \\
\hline BOAS, A. A. V. & 0.017 & HUPPES, C. M. & 0.006 \\
\hline ANDRADE, R. O. B. & 0.017 & BRANCO, L. M. P. C. & 0.006 \\
\hline OLIVIERA, E. R. & 0.017 & MACHADO, R. L. & 0.006 \\
\hline SCHRÖDER, L. M. & 0.017 & ONO, F. Z. T. & 0.006 \\
\hline HALICKI, Z. & 0.017 & STROPARO, E. J. & 0.006 \\
\hline TAKEDA, A. & 0.017 & NEIVERTH, E. M. H. B. & 0.006 \\
\hline PAULA, G. & 0.017 & FRANKLIN, R. B. & 0.006 \\
\hline PEROSA, J. M. Y. & 0.017 & FERNANDES, P. P. & 0.006 \\
\hline RECHZIEGEL, W. & 0.017 & NETO, A. F. J. & 0.006 \\
\hline BUENO, O. C. & 0.017 & & \\
\hline CRUZ, R. G. & 0.017 & & \\
\hline GAITÁN, J. A. & 0.017 & & \\
\hline
\end{tabular}

Nota: G.C - Grau de centralidade.

Fonte: Dados da pesquisa (2015). 
Tabela 3: Grau de intermediação.

\begin{tabular}{lllllc}
\hline \multicolumn{1}{c}{ AUTOR } & G. I. (\%) & \multicolumn{1}{c}{ AUTOR } & G. I. (\%) & \multicolumn{1}{c}{ AUTOR } & G .I. (\%) \\
\hline FRANCISCO, A. C.. & 0.282 & NETO, J. F. R. & 0,000 & ARRUDA, L. L. & 0,000 \\
OLIVEIRA, I. L. & 0.202 & CARMONA, C. U. M. & 0,000 & LAGIOIA, C. T. L. & 0,000 \\
IGARASHI, D. C. C. & 0.101 & CEDRAZ, K. S. A. F. & 0,000 & FRANCO, C. & 0,000 \\
SILVA, E. M. & 0.067 & PALADINI, E. P. & 0,000 & BONJOUR, S. C. M. & 0,000 \\
ALVARENGA, T. H. P. & 0.067 & SILVA, G. A. S. & 0,000 & PEREIRA, B. D. & 0,000 \\
PRADO, R. A. D. P. & 0.054 & PILATTI, L. A. & 0,000 & ABBAS, K. & 0,000 \\
MARIANO, A. M. & 0.040 & TEIXEIRA, E. B. & 0,000 & LEISMANN, E. L. & 0,000 \\
VENERAL, D. & 0.040 & EYNG, I. S.. & 0,000 & PONTES, J. & 0,000 \\
FILHO, L. P. & 0.027 & FERNANDES, P. & 0,000 & QTA, E. M. & 0,000 \\
IGARASHI, W. & 0.020 & FARIAS, T. A. & 0,000 & NETO, M. S. N. & 0,000 \\
BEURON, T. A. & 0.015 & DANGO, L. A. & 0,000 & OLIVEIRA, H. H. & 0,000 \\
CARPES, A. M. & 0.015 & RODRIGUES, J. F. & 0,000 & BISAGGIO, R. S. & 0,000 \\
\hline
\end{tabular}

Nota: G.I - Grau de intermediação. Apenas 12 autores apresentaram intermediação, os demais 161( 0,000)

Fonte: Dados da pesquisa (2015). 